Proceedings

\title{
Polymer Nanocomposites for Lowering Heating and Cooling Loads in Buildings ${ }^{+}$
}

\author{
George T. Stiubianu
}

Citation: Stiubianu, G.T. Polymer Nanocomposites for Lowering Heating and Cooling Loads in Buildings. Proceedings 2021, 69, 35. https://doi.org/10.3390/CGPM202007165

Published: 3 November 2020

Publisher's Note: MDPI stays neutral with regard to jurisdictional claims in published maps and institutional affiliations.

\section{Copyright: $₫ 2020$ by the authors.} Licensee MDPI, Basel, Switzerland. This article is an open access article distributed under the terms and conditions of the Creative Commons Attribution (CC BY) license (http://creativecommons.org/licenses/by/4.0/).
Petru Poni Institute of Macromolecular Chemistry, 700487 Iași, Romania; george.stiubianu@icmpp.ro; Tel.: +40-073-966-2129

† Presented at the First International Conference on “Green” Polymer Materials 2020, 5-25 November 2020; Available online: https://sciforum.net/conference/CGPM2020.

\begin{abstract}
Worldwide, buildings consume over $40 \%$ of the total commercial energy, and $36 \%$ of this amount is dedicated to the heating and cooling of buildings. Therefore, building environment control systems require efficient thermal management. An ideal thermal management that could lower the energy load for cooling and heating respectively would combine passive strategies for thermal control, which are characterized by low cost, straightforward implementation, and energy efficiency, with the on-demand control of heating and cooling, specific for active thermal management strategies. The scientific challenge of building an efficient platform for thermal control was addressed by using block copolymer materials in the development of nanocomposites with dynamically tunable thermal infrared properties. The polymer nanocomposites manage $60-70 \%$ of the metabolic heat flux from sedentary individuals and can modulate changes in the individual body temperature within a set-point temperature range of $8{ }^{\circ} \mathrm{C}$. This increase in the set-point temperature translates into use of air conditioning for cooling/heating with a significantly lowered load, which would further translate into a $4.3 \%$ decrease of global energy consumption.
\end{abstract}

Keywords: nanocomposite; polymer; thermal comfort; infrared radiation

\section{Introduction}

Energy consumption for building operation accounts for $\sim 40 \%$ of global energy consumption, and their heating and cooling requires $~ 36 \%$ of this amount [1]. This is an opportunity to diminish energy use worldwide [2] through the development of novel personal (localized or wearable) thermoregulatory clothing. For example, the US Department of Defense (DoD) is one of the largest energy consumers worldwide, and the 300,000 buildings spread across $>1000$ DoD installations worldwide account for $\sim 30 \%$ of DoD and $\sim 21 \%$ of USA federal government energy consumption, corresponding to an annual resource commitment of $\sim 2.1 \times 10^{14}$ Joules, at a cost of $\$ 12.5$ billion [3,4]. Worldwide, the total energy costs are $>6400$ billion US dollars [5]. There are multiple measures that can be pursued for lowering energy costs with a high return on investment, such as the retrofitting of lighting fixtures; incorporation of upgraded heating, ventilation, and air conditioning system components; adoption of novel energy distribution strategies; modification of building thermal envelopes via improved roofs and windows; and implementation of behavior-based solutions for personnel. In essence, such measures represent a strategy for transforming the resilience, reliability, and efficiency of installation energy consumption into a strong reliable method for cost-saving and efficiency improvements within the next decades. Consequently, the development of novel and improved approaches to energy management (which will facilitate the aforementioned support and refurbishing projects) remains of critical importance both for improving the energy efficiency and for enhancing the quality of life of consumers worldwide, while maintaining affordable costs for energy. 
Currently, almost all buildings, whether permanent or temporary, employ traditional systems based on decades-old thermal management methodologies, which are categorized either as "passive" or "active" depending on their mode of operation [6-9]. On the one hand, passive thermal management systems and technologies, such as building insulation and reflective coatings, leverage the intrinsic properties of their constituent materials to facilitate heat transfer via conduction, convection, and/or radiation, thereby making them static but low cost, energy efficient, and straightforward to implement [6-9]. On the other hand, active thermal management systems and technologies, such as building air conditioning and heat pumps, require the input of energy from an external source to facilitate heat transfer (via the same mechanisms), thereby making them costly, relatively inefficient, and complex, but precisely and directly controllable by a user [6-9]. Within this context, the "ideal" thermal management technology for buildings would simultaneously feature the key advantageous characteristics of both passive and active systems, i.e., a reasonable cost, little-to-no energy input requirement, straightforward implementation, and user-controlled adaptability to changing conditions.

Among passive systems, "space blankets," such as the one shown in Figure 1A, have emerged as one of the famous known thermal management technologies [10-12]. Space blankets were initially developed by NASA in the 1960s to mitigate the effect of extreme temperature fluctuations on personnel, equipment, and spacecraft, and in their most basic configuration, consist of a thin layer of metal on a transparent plastic sheet [10]. This material configuration, while highly effective at reflecting infrared radiation, features static properties and cannot be dynamically reconfigured on demand. Nonetheless, due to a favorable combination of low weight, compactness, and manufacturability, various incarnations of the space blanket have found both military and civilian applications as food packaging, emergency portable shelters, medical warming devices, protective clothing, building insulation, and solar concentrators $[11,13,14]$. Specifically, space blankets have proven particularly invaluable as emergency portable shelter components and medical warming technologies, helping to save countless lives in the field [14,15]. More generally, space blankets have made a global impact by facilitating space exploration, improving food storage, and reducing energy consumption. Despite their technological significance, space blankets have remained essentially unchanged as passive thermal management technologies and still function in much the same way as they did over fifty years ago.
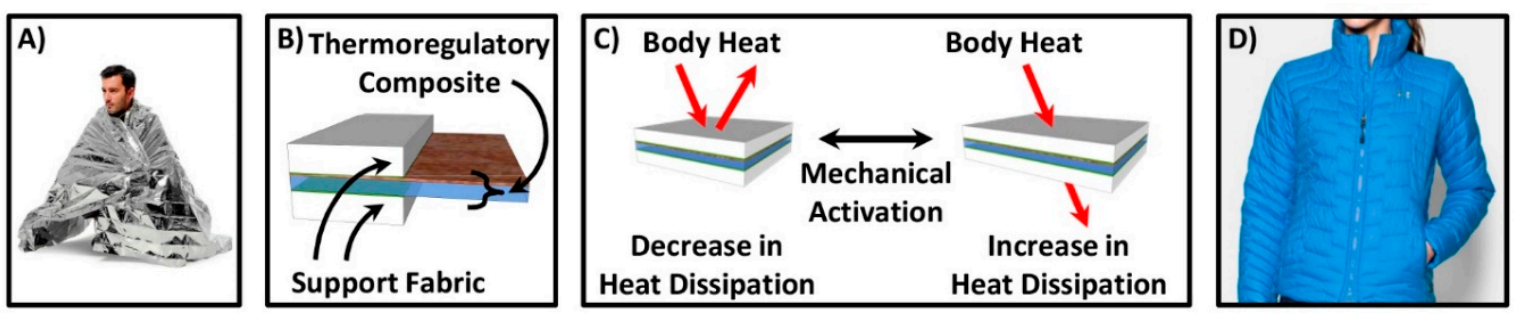

Figure 1. (A) A picture of a space blanket. (B) A schematic illustration of the proposed thermal comfort and efficiency garment. (C) A schematic of the activation mechanism for thermal efficiency nanocomposite, which shows switching between two heat dissipation states. (D) A picture of integrated thermal efficiency nanocomposite with garment.

This paper presents a laboratory technology for making wearable, clothing-integrated dual-mode (heating and cooling) polymer nanocomposites for providing thermal comfort to consumers in a passive way and benefitting the environment by lowering the energy consumption. These materials are based on thermoplastic elastomers (TPEs) coated on one side with nanometer-thick metal layer and integrated with the clothing made of well-known textile materials (such as cotton, polyester). The working principle of these materials is showed in Figure 1B,C. This material brings an opportunity to diminish energy use worldwide through the development of novel personal (localized or wearable) thermoregulatory clothing. 
The nanocomposites can be integrated in novel adaptive thermoregulatory textile as comfort garment with a multilayer structure, similar to a dynamically adaptive space blanket-like material that is sandwiched between two fabrics, as illustrated in Figure 1B. In it, the cloth would have mechanical properties similar to spandex, and wearers will induce changes in its thermoregulatory properties through mechanical activation (stretching or similar, which is easily achieved in clothing via zippering, buttoning, drawstrings, etc.). Before mechanical activation, the cloth traps infrared radiation emitted by the wearer, leading to a decrease in heat dissipation and a heating effect, and after activation, the cloth releases infrared radiation emitted by the wearer, leading to an increase in heat dissipation and a cooling effect, as illustrated in Figure 1C. Thus, garments that integrate such thermoregulating materials, such as the jacket in Figure 1D, will maintain the skin of a person wearing it at a constant temperature even when the external one varies by $>10^{\circ} \mathrm{C}$, representing a significant advance with regard to the current state-of-the-art.

The development of advanced thermal management textiles that dynamically regulate the energy exchanged by the human body with its environment is a grand challenge in the clothing industry $[16,17]$. Such technologies would allow wearers to trap or release the energy produced by their bodies via evaporation, conduction/convection, and/or radiation, and thus, would enable them to directly maintain a consistent temperature in changing thermal settings [16,17]. Within this context, the space blanket shown in Figure 1A represents the simplest incarnation of a wearable thermoregulatory technology, as it efficiently traps the body's emitted infrared radiation and lets users maintain their thermal comfort in cold environments.

\section{Experiments}

\section{Materials and Methods}

To prepare the nanosized metal layer, a $20 \mathrm{~nm}$ planar metal film (aluminium, copper, zinc, nickel) was electron-beam-evaporated onto a 6 inch diameter silicon wafer (University Wafer, Boston, MA, USA) by using electron-beam-evaporation technique (Figure 2A).

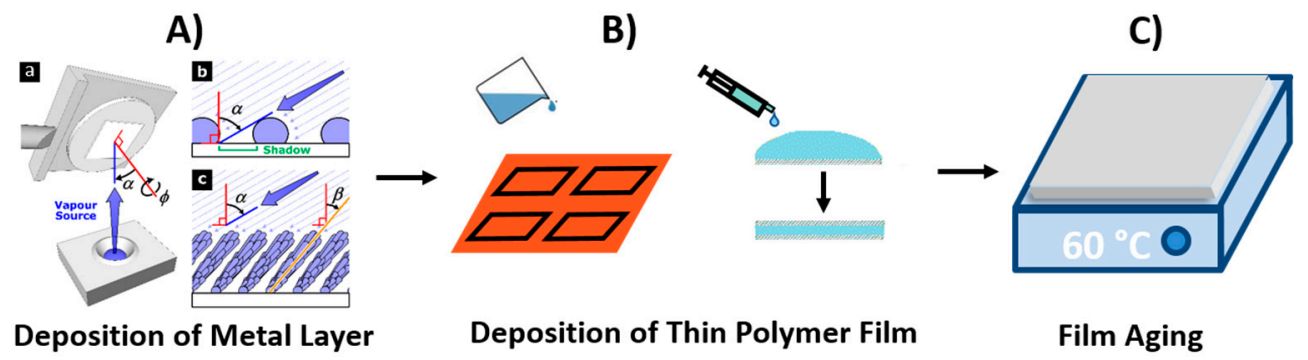

Figure 2. (A) Deposition of nanometer thick metal layer, (B) Embedding of metal layer within an infrared-transparent elastomer, (C) Maturation of nanocomposite on hot plate.

Next, to embed the nanosized metal layer within an infrared-transparent elastomer, a 10-100 micron thick film from commercially available hydrogenated styrene-based block copolymer (Kraton Polymers LLC, Belpre, OH, USA) was deposited directly onto the metal-modified substrate either by spin coating, doctor blading or dip coating (Figure 2B).

Then, the composite was maturated on a hot plate at $60-70{ }^{\circ} \mathrm{C}$ for $10-15 \mathrm{~min}$ and then delaminated from the substrate (Figure 2C). The resulting nanocomposite films were used for physical, mechanical (Instron 3365 Universal Testing System, Norwood, MA, USA), morphological (SEM), and infrared (FLIR C2 infrared video camera, Wilsonville, OR, USA) characterization experiments as needed. 


\section{Results}

The experiment began by fabricating the desired composite material according to the scheme in Figure 2A. The samples have areas of $>\sim 100 \mathrm{~cm}^{2}$ by using common laboratory techniques: spin coating, dip coating, doctor blading. The infrared-reflecting nanostructured metal film was electron-beam evaporated onto a support substrate (Figure 2A) obtaining an underlying continuous metal coating, confirmed by top-down scanning electron microscopy (SEM). Next, the metal layer was embedded in an infrared-transparent styrene-based copolymer (Figure 2B). Subsequently, the nanocomposite was aged on a hot plate and delaminated from the substrate to obtain a free-standing material, which featured a multidomain metal layer on one side, as confirmed by top-down SEM analysis. Overall, this is a robust procedure suitable for scaling for large-area nanocomposite materials.

The nanocomposites were characterized in terms of mechanical properties via tensile testing, and the engineering stress versus engineering strain data shows that such materials are soft and stretchable elastomers, with an average elastic modulus of 2-3 $\mathrm{MPa}$ and an elongation at break of $>300 \%$ (Figure 3 ). In initial state, the nanocomposite samples are opaque and highly reflective, due to coating made of metal domains. Applying a mechanical strain of only $30 \%$, the composites became partially transparent and less reflective. The soft elastomer behaviour of the nanocomposites leads to no hysteresis, with fully reversible mechanical loading/unloading in $<1$ under multiple mechanical cycles. Such characteristics make this nanocomposite a thermal switch with a fairly rapid response time.

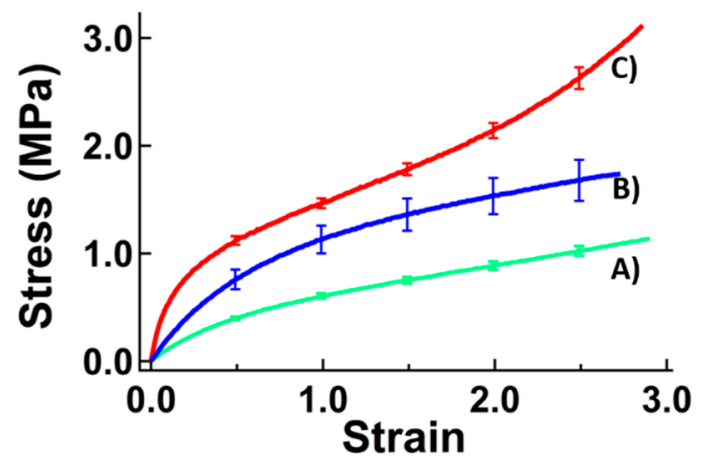

Figure 3. (A) Stress-strain curve for film of pure block copolymer with $15 \%$ styrene content, (B) Stress-strain curve for film of pure block copolymer with $30 \%$ styrene content, (C) Stress-strain curve for film made of nanocomposite material.

\section{Discussion}

The nanocomposites can modulate infrared reflectance and transmittance on demand under applied mechanical strain. In initial unstrained state (Figure 4, Left), it demonstrates infrared reflectance of close to $100 \%$ and a low average total transmittance of $<1 \%$, since the metal domains cover completely the infrared-transparent polymer matrix. When mechanical strain is applied, the infrared reflectance decreases with an increased infrared transmittance (Figure 4, Right), since the polymer matrix is only partially covered by reflecting metal nanolayer. Both the reflectance and transmittance of infrared light of the nanocomposite show linear dependence up to $50 \%$ linear mechanical strain of the samples. The ratio of infrared transmittance at strains of $0 \%$ and $100 \%$ is $>30$, showcasing the reversible thermal switching behavior.

In order to assess the reversible dynamic thermal switch capability, integrating nanocomposite and cloth were tested under mechanical actuation (Figure 5). An infrared video camera was used to record the change in temperature and the outgoing heat flux of the skin on forearms covered with screens made with integrated nanocomposite and cloth. The screen was actuated mechanically by uniaxial strain (Figure 5, bottom row). The unactuated reflective nanocomposite-based screen reflected the heat emitted by the forearm 
and raised its temperature by $\sim 1^{\circ} \mathrm{C}$ more than the same person's bare forearm. Under an applied strain of $50 \%$, the nanocomposite-based screen raised the temperature of the forearm by only $0.1^{\circ} \mathrm{C}$ more than the same person's bare forearm (10-fold reduction relative to the temperature change measured when using space blanket instead of nanocomposite integrated with cloth). Thus, the nanocomposite-based materials could be adjusted to modulate the consumers' local changes in body temperature by nearly an order of magnitude, more than the reported temperature perception thresholds of human subjects [18]. Such data open the way for research on development of nanocomposite materials that regulate the local temperature across the different parts of the human body.

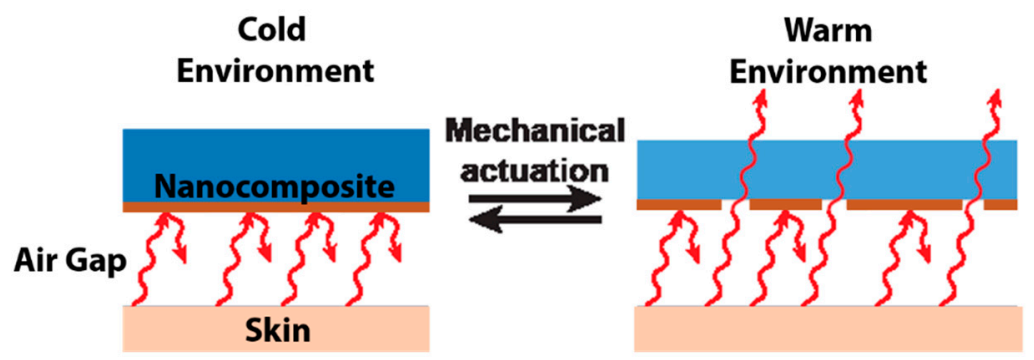

Figure 4. Schematic of the heat flux (red arrows) from human skin, relative to outside environment with a variable temperature, shown without (Left) and with (Right) mechanical actuation. After actuation, the heat flux from the skin to the environment increases, and the skin maintains a constant temperature between cooler (Left) and warmer (Right) environment temperatures.

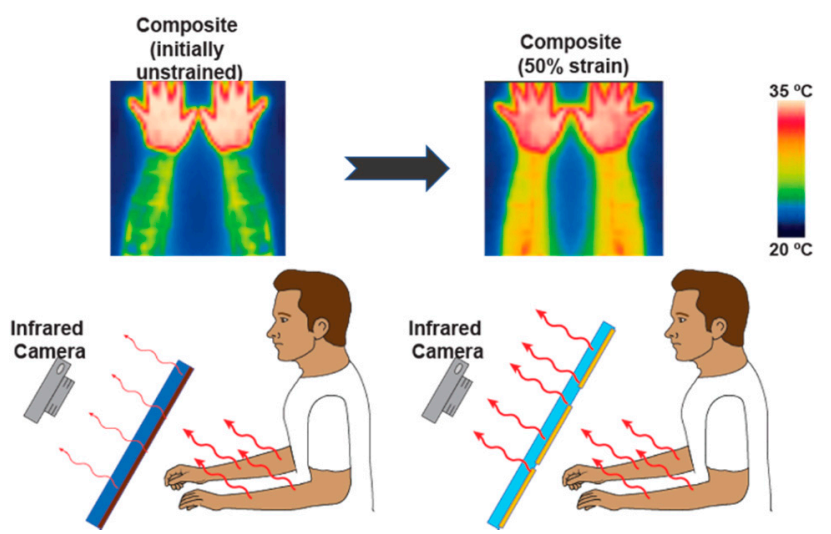

Figure 5. Test of nanocomposite cloth sleeve with infrared FLIR C2 infrared (IR) video camera: top row: IR camera image of forearms protected with screen made of nanocomposite and temperature scale; lower row: schematics of IR camera with outgoing heat flux for a human subject

The next step involved the evaluation of the nanocomposites' capability for adaptive thermal management, for possible application in clothing. The data from experimental measurements taken with the infrared camera (Figure 5) was used to model heat transfer between human skin and the surrounding environment with an air gap similar with real clothes (Figure 4). The set-point temperatures (the ones at which the body's skin temperature and outgoing heat flux remain constant, and humans maintain their thermal comfort) were calculated for multiple textile materials. The unactuated nanocomposite has a set-point temperature of $14.5^{\circ} \mathrm{C}$, similar to a space blanket $\left(14.3^{\circ} \mathrm{C}\right.$ ) (Figure 6). Applying increasingly larger mechanical actuation to the nanocomposite leads to increasing the setpoint temperature of the nanocomposite, similar to multiple common textile materials (Figure 6), and thus the nanocomposite mimics the capabilities of such fabrics and textiles. 


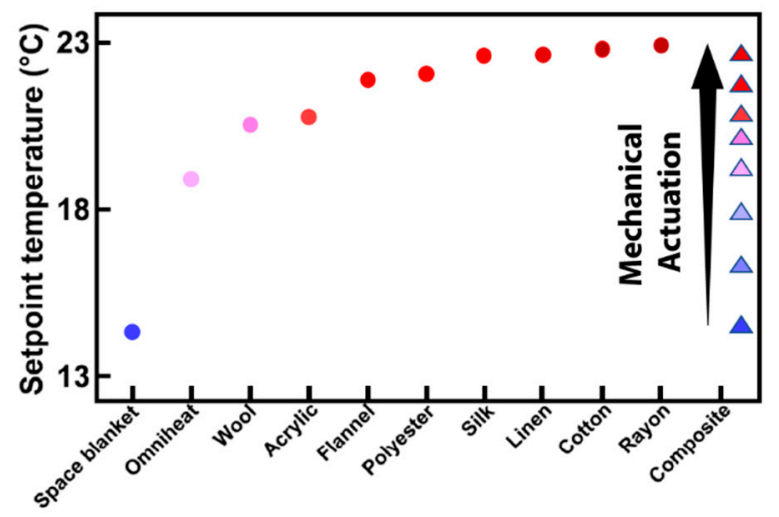

Figure 6. Environmental set-point temperatures at which a human would maintain a constant skin temperature and unchanged outgoing heat flux while wearing various common types of cloth (circles) or the nanocomposite mechanically actuated (triangles).

Any structure, either temporary or permanent, is exchanging heat with the environment (Figure 7A). The energy consumption for buildings represents $40 \%$ of worldwide energy consumed annually, and from this, $36 \%$ is used for heating and cooling (Figure 7B). The use of clothing integrating the nanocomposite would allow an expansion of the set point, similar to consumers wearing traditional thick warm clothing (Figure 6).

It is well known that expanding the cooling and heating set points by $4{ }^{\circ} \mathrm{C}$ each can lead to large energy savings up to $45 \%$ and $35 \%$, respectively [19]. Thus, the expansion of the heating set point by $4{ }^{\circ} \mathrm{C}$ (from $18{ }^{\circ} \mathrm{C}$ instead of $22^{\circ} \mathrm{C}$ ) as well as the expansion of the cooling set poit by $4{ }^{\circ} \mathrm{C}\left(26^{\circ} \mathrm{C}\right.$ instead of $\left.22^{\circ} \mathrm{C}\right)$ leads to energy savings of $35 \%$ and consequent theoretical energy savings (Figure 7B) of $0.35 * 0.36 * 0.40 * 100=5 \%$. Experimental implementation of a decrease in set-point temperature in a building led to a significant $5 \%$ decrease in energy consumption for every degree the set-point temperature was dropped [20]. Therefore, a calculation of the global impact of decreasing the set-point temperature from $22{ }^{\circ} \mathrm{C}$ to $16{ }^{\circ} \mathrm{C}\left(6^{\circ} \mathrm{C}\right.$ difference) would yield $5 \% /{ }^{\circ} \mathrm{C} * 6{ }^{\circ} \mathrm{C}=30 \%$ reduction in energy consumption in buildings. Therefore, personal clothing with integrated nanocomposite which provides an expanded range of thermal comfort temperatures and thermal efficiency for users holds the potential to reduce global energy consumption by up to $0.30 * 0.36 * 0.40 \sim 4 \%$.
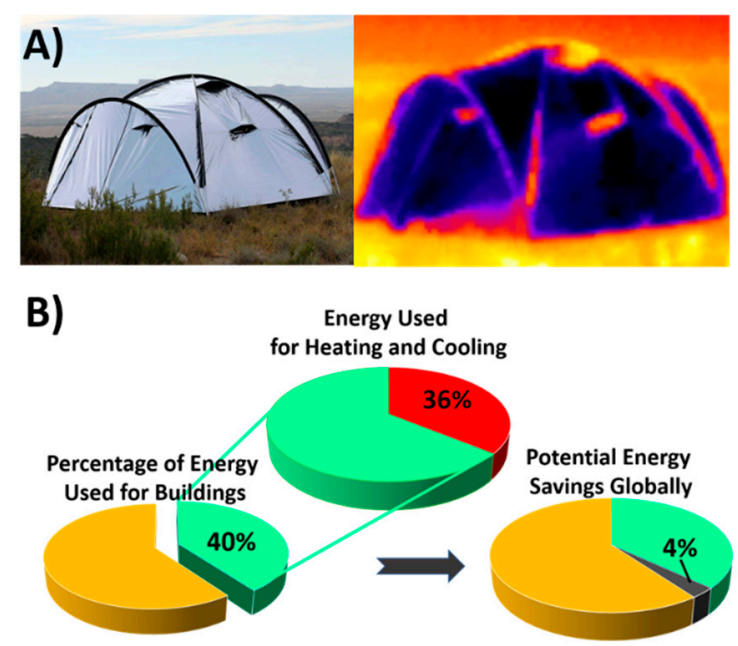

Figure 7. (A) Left: visible image of tent. Right: infrared camera of same tent, showing heat losses, (B) Ratio of worldwide energy consumed for buildings, and ratio of energy consumed for heating and cooling buildings [21]. 


\section{Conclusions}

The nanocomposite described here can be integrated with textile cloth for development of an artificial thermoregulatory platform. The nanocomposite functions via a unique mechanism that relies on reversible and mechanically actuated changes in surface microstructure. Such materials change their reflectance and transmittance in the infrared region of the electromagnetic spectrum and their thermoregulatory properties resemble those of common materials, such as the space blanket, fleece lining, wool, and cotton. The nanocomposite behaves like a radiative thermal switch with straightforward mechanical actuation method without hysteresis, and can regulate skin temperature changes for consumers in real time. Even more, such nanocomposites can be manufactured from low-cost commercially available starting materials using scalable processes.

Institutional Review Board Statement: Ethical review and approval were waived for this study, due to no invasive procedures being carried on human test subject.

Informed Consent Statement: Not applicable.

Data Availability Statement: Data available on request due to restrictions e.g., privacy or ethical. The data presented in this study are available on request from the corresponding author. The data are not publicly available due to privacy, as the data belongs to the author of the study.

Acknowledgments: This work was supported by a grant from the Romanian National Authority for Scientific Research and Innovation, CNCS/CCCDI -UEFISCDI, Smart composite system with self-controlled configuration developed from shape memory/amorphous magnetic materials in elastomeric matrices-SMAMEM, cod project: PN-III-P2-2.1-PED-2019-4138, Contract 321PED/2020.

Conflicts of Interest: The author declares no conflict of interest.

\section{References}

1. Ürge-Vorsatz, D.; Cabeza, L.F.; Serrano, S.; Barreneche, C.; Petrichenko, K. Heating and cooling energy trends and drivers in buildings. Renew. Sustain. Energy Rev. 2015, 41, 85-98, doi:10.1016/j.rser.2014.08.039.

2. U.S. Department of Energy Advanced Research Projects Agency. DELTA Program Overview. 2014. Available online: https://arpa-e.energy.gov/?q=site-page/delta-kickoff-meeting (accessed on 4 September 2019).

3. Conger, J. Department of Defense Annual Energy Management Report Fiscal Year 2016; Office of the Assistant Secretary of Defense, U.S. Government Printing Office: Washington, DC, USA, 2017.

4. Army Net Zero Page. Assistant Secretary of the Army for Installations, Energy, and Environment. Available online: http://www.asaie.army.mil/Public/ES/netzero/ (accessed on 4 September 2019).

5. World Energy Expenditures. Enerdata. Available online: https://www.enerdata.net/publications/executive-briefing/world-energy-expenditures.html (accessed on 4 September 2019).

6. Wang, Y.; Shukla, A.; Liu, S. A state of art review on methodologies for heat transfer and energy flow characteristics of the active building envelopes. Renew. Sustain. Energy Rev. 2017, 78, 1102-1116.

7. Gupta, N.; Tiwari, G.N. Review of passive heating/cooling systems of buildings. Energy Sci. Eng. 2016, 4, 305-333.

8. Grondzik, W.; Furst, R. HVAC Components and Systems. In Vital Signs Curriculum Materials Project; Florida A\&M University: Tallahassee, FL, USA, 2000.

9. Dincer, I.; Rosen, M. Thermal Energy Storage, Systems and Application; John Wiley \& Sons: Chichester, UK, 2002.

10. Giaimo, C. The Weird History of the Space Blanket. Atlas Obscura. Available online: http://www.atlasobscura.com/articles/theweird-history-of-the-space-blanket (accessed on 3 September 2019).

11. Reflecting on Space Benefits: A Shining Example. NASA Spinoff. Available online: http://spinoff.nasa.gov/Spinoff2006/ch_9.html (accessed on 3 September 2019).

12. Ronca, D. How Space Blankets Work. HowStuffWorks.com. Available online: http://adventure.howstuffworks.com/survival/gear/space-blanket.htm (accessed on 3 September 2019).

13. 6 Applications for Heat Reflective Fabric. Sigma Labs. Available online: http://www.sigmalabs.com/blog/6-applications-forheat-reflective-fabric (accessed on 3 September 2019).

14. Roussey, B. The 10 Best Survival Blankets for Harsh Weather. Survivor's Fortress. Available online: http://survivorsfortress.com/best-survival-blankets/ (accessed on 3 September 2019).

15. John, M.; Ford, J.; Harper, M. Peri-operative warming devices: Performance and clinical application. Anaesthesia 2014, 69, 623638.

16. Song, G. Improving Comfort in Clothing; Elsevier: Cambridge, UK, 2011.

17. Yazdi, M.M.; Sheikhzadeh, M. Personal cooling garments: A review. J. Text. Inst. 2014, 105, 1231-1250.

18. Stevens, J.C.; Choo, K.K. Temperature sensitivity of the body surface over the life span. Somatosens. Mot. Res. 1998, 15, 13-28. 
19. Hoyt, T.; Lee, K.H.; Zhang, H.; Arens, E.; Webster, T. Energy savings from extended air temperature setpoints and reductions in room air mixing. In Proceedings of the International Conference on Environmental Ergonomics 2009, Boston, MA, USA, 2-7 August 2009; Curran Associates Inc.: Red Hook, NY, USA, 2011; pp. 608-612.

20. Saurav, K.; Jain, M.; Bandhyopahyay, S. Reducing energy consumption for space heating by changing zone temperature: Pilot trial in Luleå, Sweden. In Proceedings of the Ninth International Conference on Future Energy Systems, Karlsruhe, Germany, 12-15 June 2018; pp. 266-270.

21. International Energy Agency. Energy Technology Perspectives 2017; International Energy Agency: Paris, France, 2017. 